\title{
An Analytical Solution for Queue: M/D/1 with Balking
}

\author{
Kotb Abdel Hamid Kotb, Moamer Akhdar* \\ Department of Mathematics and Statistics, Faculty of Science, Tanta University, Tanta, Egypt \\ Email address: \\ msukss2010@gmail.com (K. A. H. Kotb), msukss2010@yahoo.com (M. Akhdar) \\ ${ }^{*}$ Corresponding author
}

\section{To cite this article:}

Kotb Abdel Hamid Kotb, Moamer Akhdar. An Analytical Solution for Queue: M/D/1 with Balking. Applied and Computational Mathematics. Vol. 7, No. 2, 2018, pp. 31-39. doi: 10.11648/j.acm.20180702.11

Received: January 17, 2018; Accepted: January 31, 2018; Published: February 27, 2018

\begin{abstract}
In this paper we examine the how to of deriving analytical solution in steady-state for non-truncated single-server queueing and service time are fixed (deterministic) with addition the concept balking, using iterative method and the probability generating function. Some measures of effecting of queuing system are obtained using a smooth and logical manner also some special cases of this system. Finality, some numerical values are given showily the effect of correlation between the $\left(p_{0}, p_{n}, L, W_{q}\right)$ and the additional concepts.
\end{abstract}

Keywords: Deterministic, Queueing System, Measures of Effectiveness, Generating Function

\section{Introduction}

The queueing M/D/1 of queues that did not taking the right of study, especially when adding some concepts of loss of impatient. Oliver [1] in 1968 he studied the waiting time distribution for the constant service queue $(\mathrm{M} / \mathrm{D} / 1)$. Iversen [2] studied exact calculation of waiting time distributions in queueing systems with constant holding times. Iversen and Staalhagen [3] in 1999 he studied waiting time distribution in queue $\mathrm{M} / \mathrm{D} / 1$. Brun and Garcia [4] derived an analytical solution of finite capacity for queue M/D/1. Koba [5] search Stability condition for $\mathrm{M} / \mathrm{D} / 1$ retrial queuing system with a limited waiting time. Also Koba [6] in 2000 studied the An $\mathrm{M} / \mathrm{D} / 1$ queuing system with partial synchronization of its incoming flow and demands repeating at constant intervals. A series expansion for the stationary probabilities of an $\mathrm{M} / \mathrm{D} / 1$ queue is obtained by Nakagawa [7]. And, Prasad and Usha [10] in 2015 studied comparison between M/M/1 and $\mathrm{M} / \mathrm{D} / 1$ queuing models to vehicular traffic at Kannyakumari district. Other related studies are presented by Hussain et al. [11], Kim and Kim [12] and Baek et al. [13]. Recently, Kotobi and Bilén [14] focused Spectrum sharing via hybrid cognitive players evaluated by an $\mathrm{M} / \mathrm{D} / 1$ queuing model.

In this paper, we have proposed analytical solution of the steady-state in the non-truncated single-channel Markovian queue $\mathrm{M} / \mathrm{D} / 1$ subject to balking. The probability that there are $\mathrm{n}$ customers in the system, the probability of empty system and some measures of effectiveness are obtained using iterative method, probability generating function. Some special cases are deduced. Finally, a simulation study has been considered to illustrate the numerical application for the model.

\section{Basic Notations and Assumptions}

To construct the system of this paper, we define the following parameters:

$P(z)=$ The Probability generating function.

$p_{n}=$ Stead-state probability that there are $n$ customers in the system.

$\lambda=$ Mean arrival rate.

$\mu=$ Mean service rate.

$D=$ The fixed time of service between each customer and the other.

$n=$ Number of customers in the system.

$\beta=$ The probability that the customer joins the queue.

$\rho=\lambda D=$ Utilization factor.

$L=$ Expected number of customers in the system.

$L_{q}=$ Expected number of customers waiting to be served.

$W=$ Expected waiting time in the system. 
$L_{q}=$ Expected waiting time in the queue.

The assumptions of this model are listed as follows:

(1) Customers arrive at the server one by one according to Poisson process with rate $\lambda$. Assume $(1-\beta)$ be the probability that a customer balks, $0 \leq \beta<1, n \geq 1$; and $\beta=1, n=0$. Thus it is clear that:

$$
\lambda_{n}=\left\{\begin{array}{l}
\lambda, n=0 \\
\beta \lambda, n \geq 1
\end{array}\right.
$$

(2) Service times of the customers are deterministic time D with rate $\mu$, where

$$
\mu=\left\{\begin{array}{lc}
0, & \text { no service } \\
1, & \text { timer unit }
\end{array}\right.
$$

(3) A single server serves entities one at a time from the front of the queue, according to a first-come, firstserved discipline. When the service is complete the entity leaves the queue and the number of entities in the system reduces by one.

(4) The buffer is of infinite size, so there is no limit on the number of entities it can contain.

\section{Model Formulation and Analysis}

Due to the lack of a Poisson condition for the server, it is using the equation a no degenerate solution to the stationary to find $p_{n}$, where

$$
\left\{\begin{array}{l}
p=p A \\
p e=1
\end{array}\right.
$$

with

$$
\begin{gathered}
p=\left(p_{n}\right)_{1 . n}=\left(\begin{array}{lllll}
p_{0} & p_{1} & p_{2} & \ldots & p_{n}
\end{array}\right), A=\left(a_{n}\right)_{n . n} \text { and } \\
a_{n}= \begin{cases}e^{-\lambda} & , n=0 \\
\frac{e^{-\beta \lambda}(\beta \lambda)^{n}}{n !}, & n \geq 1\end{cases}
\end{gathered}
$$

Then

$$
A=\left(\begin{array}{ccccc}
a_{0} & a_{1} & a_{2} & a_{3} & \ldots \\
a_{0} & a_{1} & a_{2} & a_{3} & \ldots \\
0 & a_{0} & a_{1} & a_{2} & \ldots \\
0 & 0 & a_{0} & a_{1} & \ldots \\
0 & 0 & 0 & a_{0} & \ldots \\
. . & . . & . . & . . & \ldots
\end{array}\right)
$$

From equations (1), (2) and (3), we get:

$$
\begin{gathered}
p_{0}=e^{-\lambda} p_{0}+e^{-\lambda} p_{1} \\
p_{1}=\beta \lambda e^{-\beta \lambda} p_{0}+\beta \lambda e^{-\beta \lambda} p_{1}+e^{-\lambda} p_{2}
\end{gathered}
$$

Thus

$$
p_{n}=\sum_{i=1}^{n} \frac{(\beta \lambda)^{i} e^{-\beta \lambda}}{i !} p_{n-i+1}+\frac{(\beta \lambda)^{n} e^{-\beta \lambda}}{n !} p_{0}+e^{-\lambda} p_{n+1}, \quad n \geq 1
$$

To be finding explicit $p_{0}$ in $\lambda$, we use the probability generating function $P(z)$ whereas:

$$
P(z)=\sum_{n=0}^{\infty} p_{n} z^{n} \text { and } a(z)=\sum_{n=0}^{\infty} a_{n} z^{n}
$$

Multiplying each equation (4), (5), (6) and (8) by the appropriate power of z, we obtain:

$$
z p_{0}=a_{0} p_{0} z+a_{0} p_{1} z
$$




$$
\begin{gathered}
p_{1} z^{2}=a_{1} p_{0} z^{2}+a_{1} p_{1} z^{2}+a_{0} p_{2} z^{2} \\
p_{2} z^{3}=a_{2} p_{0} z^{3}+a_{2} p_{1} z^{3}+a_{1} p_{2} z^{3}+a_{0} p_{3} z^{3} \\
p_{n} z^{n+1}=p_{0} a_{n} z^{n+1}+p_{1} a_{n} z^{n+1}+p_{2} a_{n-1} z^{n+1}+p_{3} a_{n-2} z^{n+1}+\ldots
\end{gathered}
$$

Taking $\sum_{n=0}^{\infty}$ into equation (14), we get:

$$
z \sum_{n=0}^{\infty} p_{n} z^{n}=p_{0} z \sum_{n=0}^{\infty} a_{n} z^{n}+p_{1} z \sum_{n=0}^{\infty} a_{n} z^{n}+p_{2} z^{2} \sum_{n=1}^{\infty} a_{n-1} z^{n-1}+p_{3} z^{3} \sum_{n=2}^{\infty} a_{n-2} z^{n-2}+\ldots
$$

From (10) and (15), obtain as:

$$
P(z)=\frac{p_{0}(1-z) a(z)}{a(z)-z}
$$

Substituting equation (10) into (16), we find:

$$
P(z)=\frac{p_{0}(1-z)\left(\sum_{n=1}^{\infty} \frac{(\beta \lambda)^{n} e^{-\beta \lambda}}{n !}+e^{-\lambda}\right)}{\sum_{n=1}^{\infty} \frac{(\beta \lambda)^{n} e^{-\beta \lambda}}{n !}+e^{-\lambda}-z}
$$

Thus

$$
P(z)=\frac{p_{0}(1-z)\left(e^{-\beta \lambda(1-z)}+e^{-\lambda}-e^{-\beta \lambda}\right)}{\left(e^{-\beta \lambda(1-z)}+e^{-\lambda}-e^{-\beta \lambda}\right)-z}
$$

Multiplying numerator and the denominator of the equation (17) in $\left(e^{-\beta \lambda(1-z)}+e^{-\lambda}-e^{-\beta \lambda}\right)^{-1}$, we obtain:

$$
P(z)=\frac{p_{0}(1-z)}{1-z\left(e^{-\beta \lambda(1-z)}+e^{-\lambda}-e^{-\beta \lambda}\right)^{-1}}
$$

Using the fact that $P(1)=1$, along with LHopitals rule, we find:

$$
p_{0}=\left(1+e^{-\lambda}-e^{-\beta \lambda}\right)^{-1}\left[1-\beta \lambda\left(1+e^{-\lambda}-e^{-\beta \lambda}\right)^{-1}\right]
$$

\section{Measures of Effectiveness}

To calculate the expected number of units in the system, using as:

$$
L=E(n)=\sum_{n=0}^{\infty} n p_{n}
$$

Consider

$$
A=\sum_{n=0}^{\infty} n^{2} p_{n}
$$

From equation (9) and (22), we find:

$$
A=\sum_{n=1}^{\infty} n^{2} \sum_{i=0}^{n} \frac{e^{-\beta \lambda}(\beta \lambda)^{i}}{i !} p_{n-i+1}+\sum_{n=1}^{\infty} n^{2} \frac{(\beta \lambda)^{n} e^{-\beta \lambda}}{n !} p_{0}+e^{-\lambda} \sum_{n=1}^{\infty} n^{2} p_{n+1}
$$

with

$$
B=\sum_{n=0}^{\infty} n^{2} \sum_{i=0}^{n} \frac{e^{-\beta \lambda}(\beta \lambda)^{i}}{i !} p_{n-i+1} \text { and } C=\sum_{n=1}^{\infty} n^{2} \frac{(\beta \lambda)^{n} e^{-\beta \lambda}}{n !} p_{0}
$$

From equation (24) and same algebra, we get:

$$
C=\beta \lambda p_{0}(1+\beta \lambda)
$$

and

$$
B=\sum_{i=0}^{\infty} \frac{e^{-\beta \lambda}(\beta \lambda)^{i}}{i !} \sum_{m=1}^{\infty} m^{2} p_{m}+2 \sum_{i=0}^{\infty} \frac{i e^{-\beta \lambda}(\beta \lambda)^{i}}{i !} \sum_{m=1}^{\infty} m p_{m}-2 \sum_{i=0}^{\infty} \frac{e^{-\beta \lambda}(\beta \lambda)^{i}}{i !} \sum_{m=1}^{\infty} m p_{m}
$$




$$
+\sum_{i=0}^{\infty} \frac{i^{2} e^{-\beta \lambda}(\beta \lambda)^{i}}{i !} \sum_{m=1}^{\infty} p_{m}-2 \sum_{i=0}^{\infty} \frac{i e^{-\beta \lambda}(\beta \lambda)^{i}}{i !} \sum_{m=1}^{\infty} p_{m}+\sum_{i=0}^{\infty} \frac{e^{-\beta \lambda}(\beta \lambda)^{i}}{i !} \sum_{m=1}^{\infty} p_{m}
$$

From equation (21), (22), (25) and (26), we find:

$$
L=\frac{\beta \lambda(1+\beta \lambda)+(1-2 \beta \lambda)\left(1-p_{0}\right)}{2(1-\beta \lambda)},
$$

Also, calculate the expected number of units in the queue, using as:

$$
L_{q}=L-\left(1-p_{0}\right)
$$

So, calculate the expected waiting time in the system, using as:

$$
W=L / \lambda
$$

And, Calculate expected waiting time in the queue, using as:

$$
W_{q}=L_{q} / \lambda
$$

Where

$p_{0}=\left(1+e^{-\lambda}-e^{-\beta \lambda}\right)^{-1}\left[1-\beta \lambda\left(1+e^{-\lambda}-e^{-\beta \lambda}\right)^{-1}\right]$

\section{Special Cases}

Some queuing systems can be obtained as special cases of this system:

Case (1): Let $\beta=1$, this is the queue: $\mathrm{M} / \mathrm{D} / 1$ without any concepts. Then relations (9), (20), (27), (28), (29) and (30) are expressed as:

The steady-seat probability that there $n$ customers in the system is:

$$
p_{n}=\sum_{i=0}^{n} \frac{\lambda^{i} e^{-\lambda}}{i !} p_{n-i+1}+\frac{\lambda^{n} e^{-\lambda}}{n !} p_{0}, \quad n \geq 0,
$$

The steady-seat probability that there are no customers in the system is:

$$
p_{0}=1-\lambda
$$

The expected number of customers in the system is:

$$
L=\rho+\frac{1}{2}\left(\frac{\rho^{2}}{1-\rho}\right),
$$

The expected number of units in the queue is:

$$
L_{q}=\frac{1}{2}\left(\frac{\rho^{2}}{1-\rho}\right),
$$

The expected waiting time in the system is:

$$
W=1+\frac{1}{2}\left(\frac{\rho}{1-\rho}\right)
$$

And the expected waiting time in the queue is:

$$
W_{q}=\frac{1}{2}\left(\frac{\rho}{1-\rho}\right)
$$

where $\rho=\lambda$

Relations (32-37) are the same results as Harris [9], Brun and Garcia [4] and Iversen [2].

Case (2): Let $\beta=1$ and service times of the customers are exponential random variables with rate $\mu_{n}=\mu$, this is the queue: $\mathrm{M} / \mathrm{M} / 1$ without any concepts. Then relations (20), (27), (28), (29) and (30) are expressed as:

The steady-seat probability that there are no customers in the system is:

$$
p_{0}=1-\rho,
$$

The expected number of customers in the system is:

$$
L=\frac{\rho}{1-\rho},
$$

The expected number of units in the queue is:

$$
L_{q}=\frac{\rho^{2}}{1-\rho},
$$

The expected waiting time in the system is:

$$
W=\frac{1}{\lambda}\left(\frac{\rho}{1-\rho}\right),
$$

And the expected waiting time in the queue is:

$$
W_{q}=\frac{1}{\lambda}\left(\frac{\rho^{2}}{1-\rho}\right)
$$

where $\rho=\lambda / \mu$

Relations (38-42) are the same results as Harris [9], Prasad and Usha [10].

\section{An Illustrative Example}

The results of $p_{0}$ and $L$ for different values of $\beta$ and $\lambda$ are shown in the following table1: 
Table 1. The results of $p_{0}$ and $L$.

\begin{tabular}{llll}
\hline $\boldsymbol{\beta}$ & $\boldsymbol{\lambda}$ & $\boldsymbol{p O}_{\boldsymbol{O}}$ & $\boldsymbol{L}$ \\
\hline 0.150 & 0.050 & 0.999 & 0.004 \\
0.300 & 0.200 & 0.923 & 0.092 \\
0.450 & 0.350 & 0.872 & 0.160 \\
0.600 & 0.500 & 0.755 & 0.349 \\
0.750 & 0.650 & 0.510 & 0.720 \\
0.880 & 0.780 & 0.295 & 1.430 \\
0.950 & 0.900 & 0.130 & 3.340 \\
\hline
\end{tabular}

Solution of the model may be determined more readily by plotting $p_{0}$ against $\beta$ and $\lambda$ as shown in Figure 1. Also $L$ is drawn against $\beta$ and $\lambda$ as given in Figure 2 .

As we can see in figure 1 , shows that the increased the both of (arrival rate and Balking) offset it decrease the probability that there are no customers in the system. It is seen in figure 2; shows that the increased the both of (arrival rate and Balking) offset it increase the expected number of customers in the system.

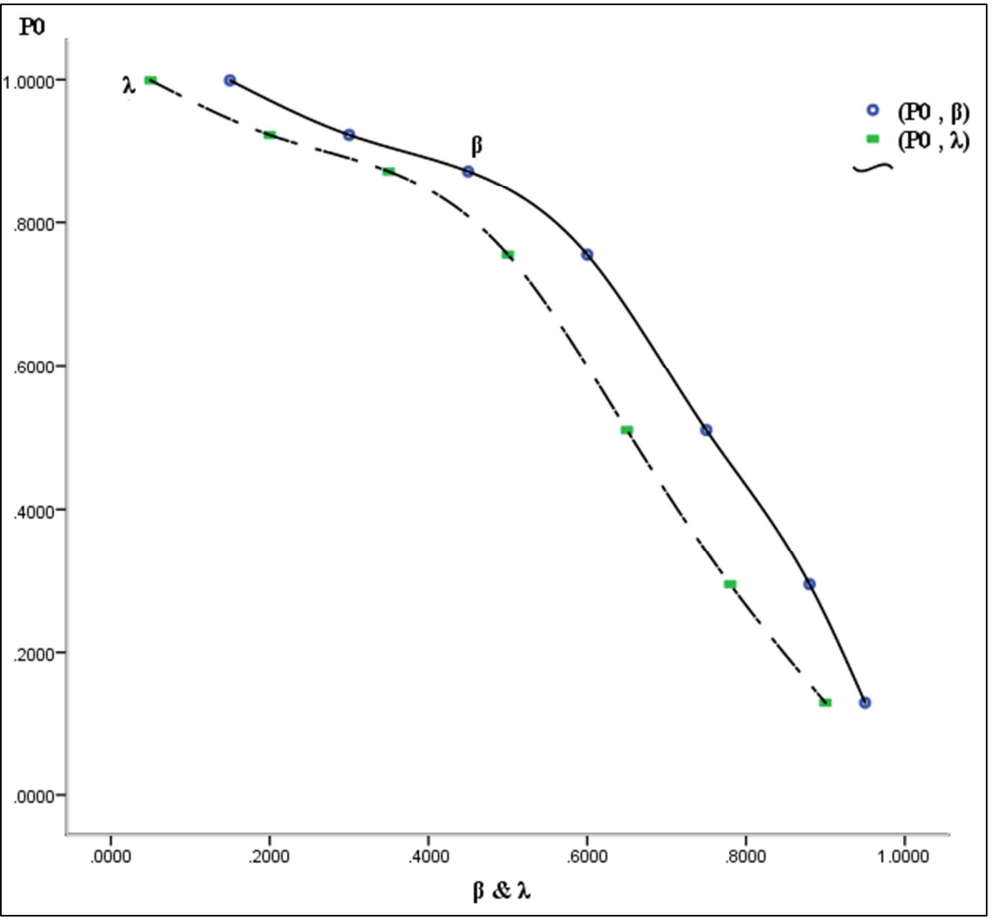

Figure 1. The relation between $p_{0} \&(\beta$ and $\lambda)$

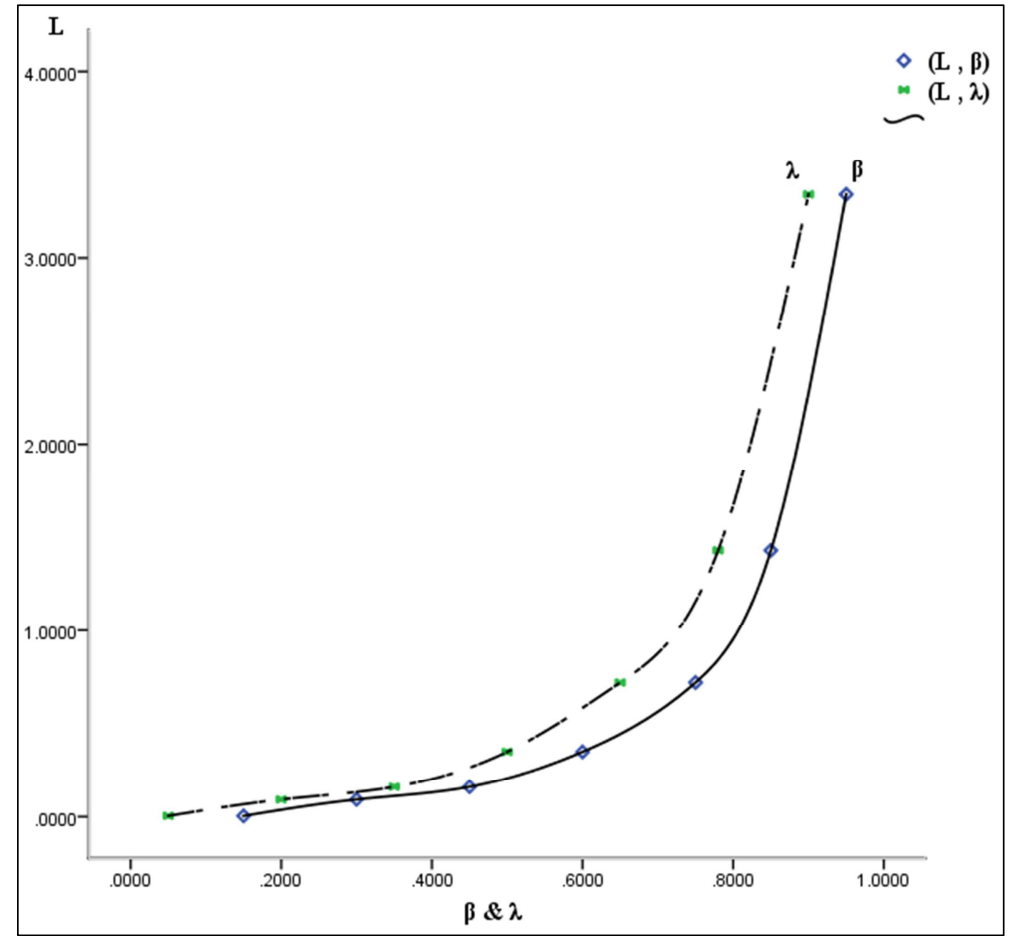

Figure 2. The relation between $L \&(\beta$ and $\lambda)$ 
Also, assume the $n=3$ units. The results of $p_{0}$, $p_{1}, p_{2}, p_{3}, L$ and $W_{q}$ for different values of $\lambda$ are shown in the following table 2 :

Table 2. The results of $p_{0}$ and $L$ without any concepts.

\begin{tabular}{ccccccc}
\hline $\boldsymbol{\lambda}$ & $\boldsymbol{p}_{\boldsymbol{O}}$ & $\boldsymbol{p}_{\boldsymbol{1}}$ & $\boldsymbol{p}_{\boldsymbol{2}}$ & $\boldsymbol{p}_{\boldsymbol{3}}$ & $\boldsymbol{L}$ & $\boldsymbol{W}_{\boldsymbol{q}}$ \\
\hline 0.1 & 0.9 & 0.05 & 0.01 & 0.0002 & 0.11 & 0.060 \\
0.2 & 0.8 & 0.18 & 0.02 & 0.0020 & 0.23 & 0.125 \\
0.3 & 0.7 & 0.24 & 0.05 & 0.0070 & 0.36 & 0.214 \\
\hline
\end{tabular}

\begin{tabular}{ccccccc}
\hline $\boldsymbol{\lambda}$ & $\boldsymbol{p}_{\boldsymbol{0}}$ & $\boldsymbol{p}_{\boldsymbol{1}}$ & $\boldsymbol{p}_{\boldsymbol{2}}$ & $\boldsymbol{p}_{\boldsymbol{3}}$ & $\boldsymbol{L}$ & $\boldsymbol{W}_{\boldsymbol{q}}$ \\
\hline 0.5 & 0.5 & 0.32 & 0.12 & 0.0400 & 0.75 & 0.500 \\
0.7 & 0.3 & 0.30 & 0.18 & 0.1000 & 1.52 & 1.170 \\
0.8 & 0.2 & 0.25 & 0.19 & 0.1300 & 2.40 & 2.000 \\
0.9 & 0.1 & 0.15 & 0.14 & 0.1150 & 5.00 & 4.500 \\
\hline
\end{tabular}

Solution of the model may be determined more readily by plotting $p_{0}, P_{1}, P_{2}, p_{3}, L$ and $W_{q}$ against $\lambda$ as given in Figures $3,4,5,6,7$ and 8 respectively.

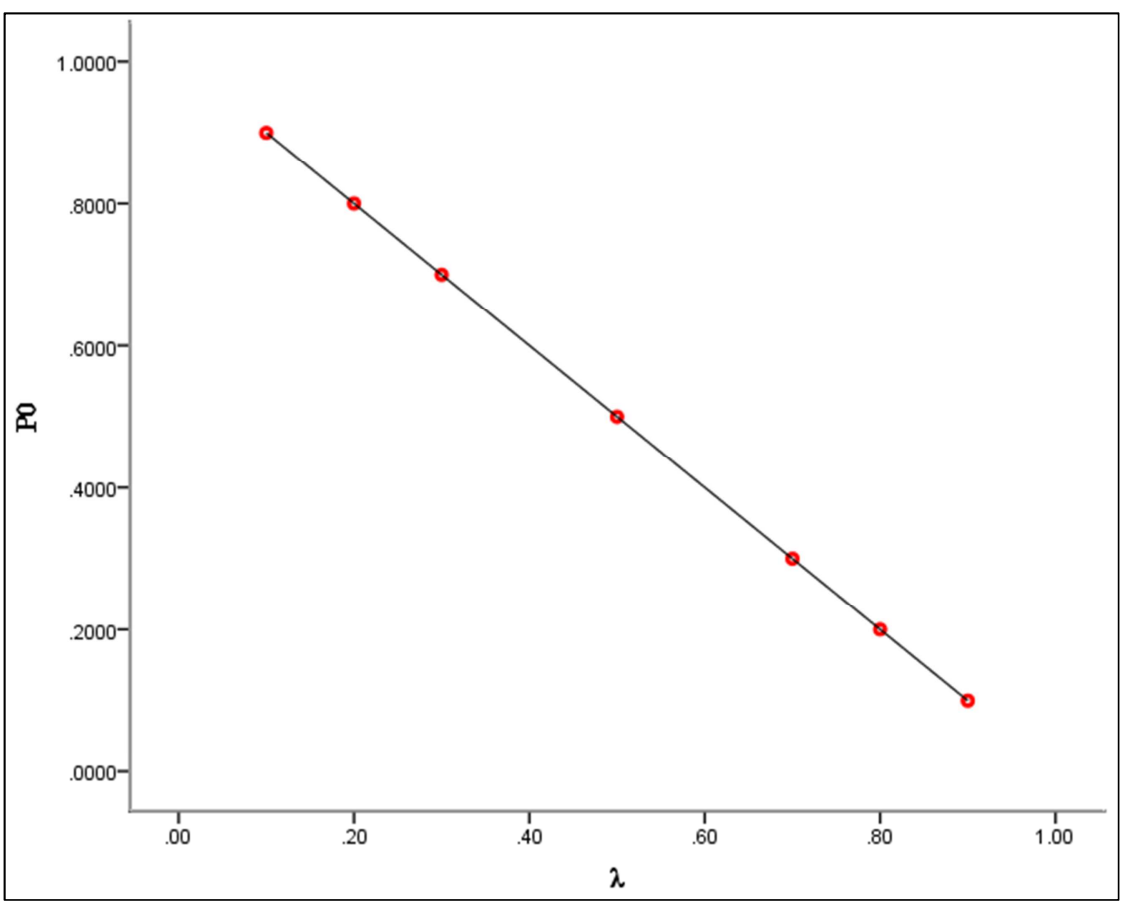

Figure 3. The relation between $p_{0} \& \lambda$.

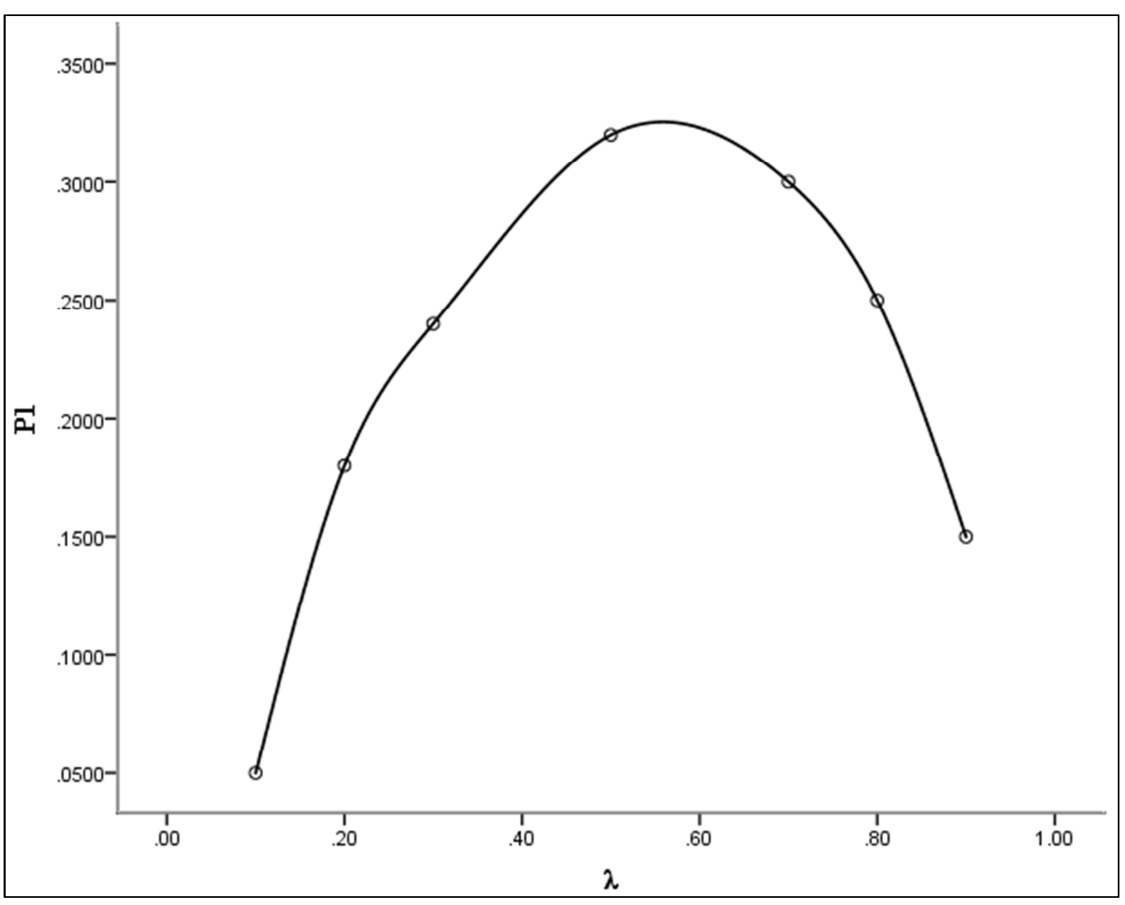

Figure 4. The relation between $p_{1} \& \lambda$. 


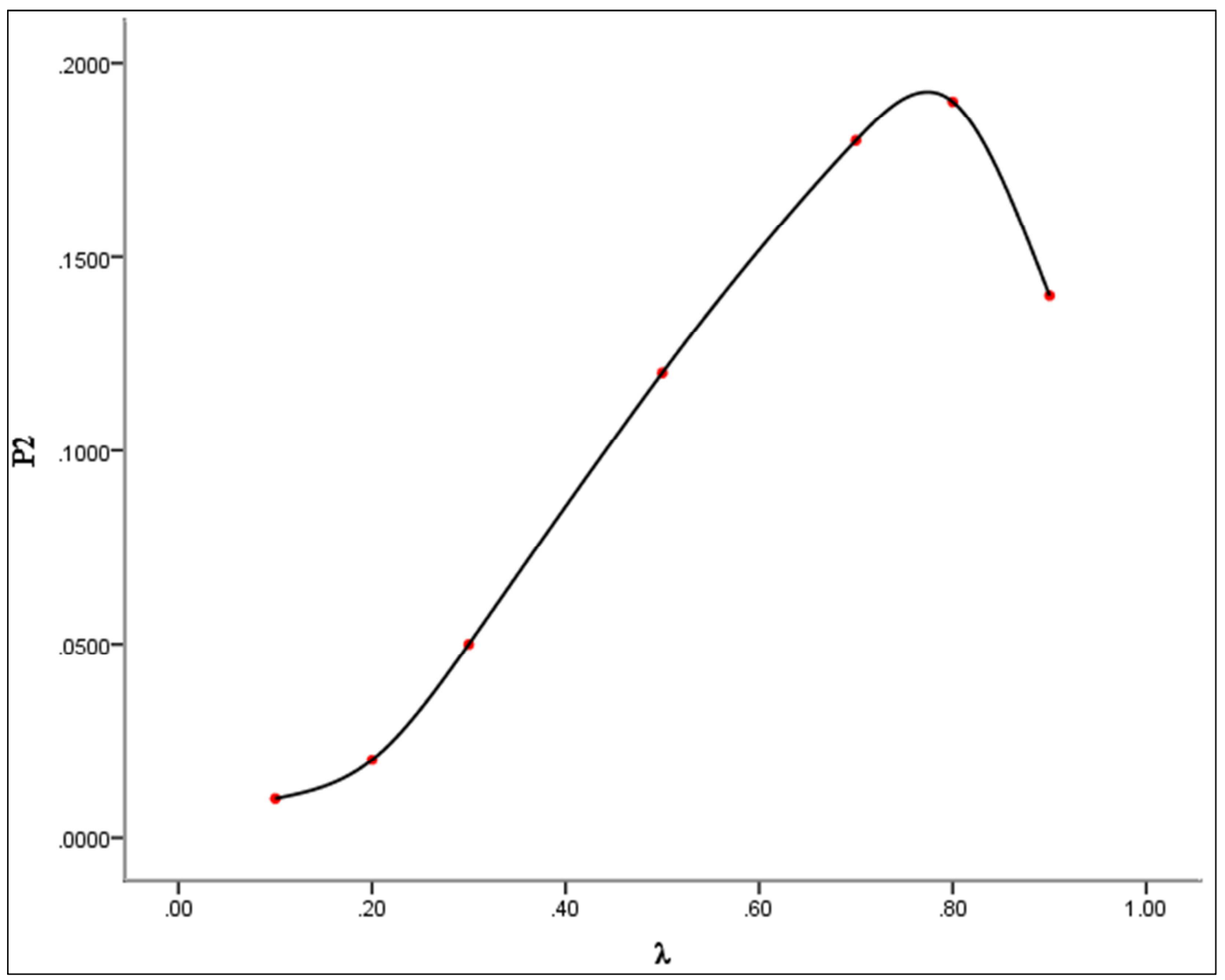

Figure 5. The relation between $p_{2} \& \lambda$

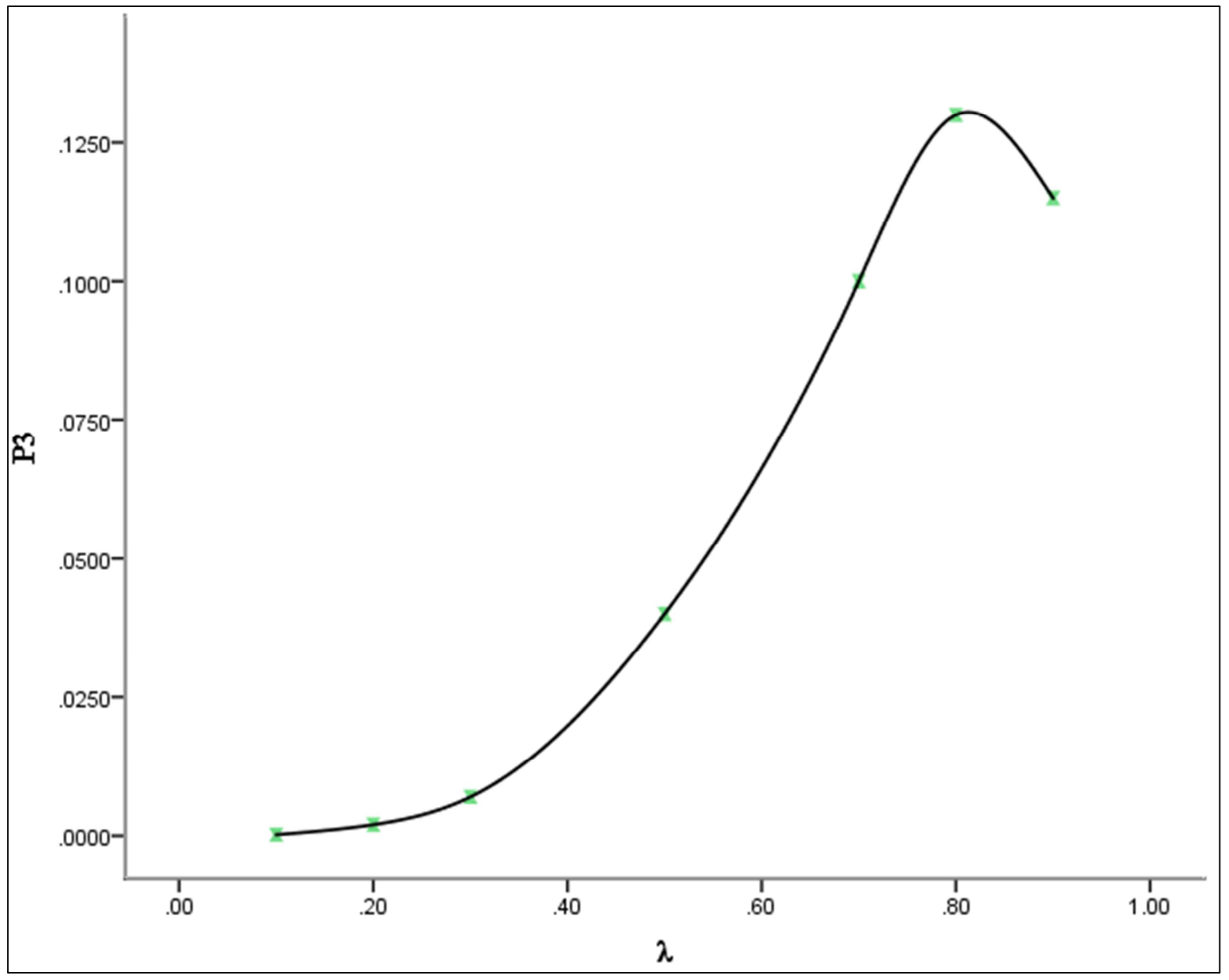

Figure 6. The relation between $p_{3} \& \lambda$. 


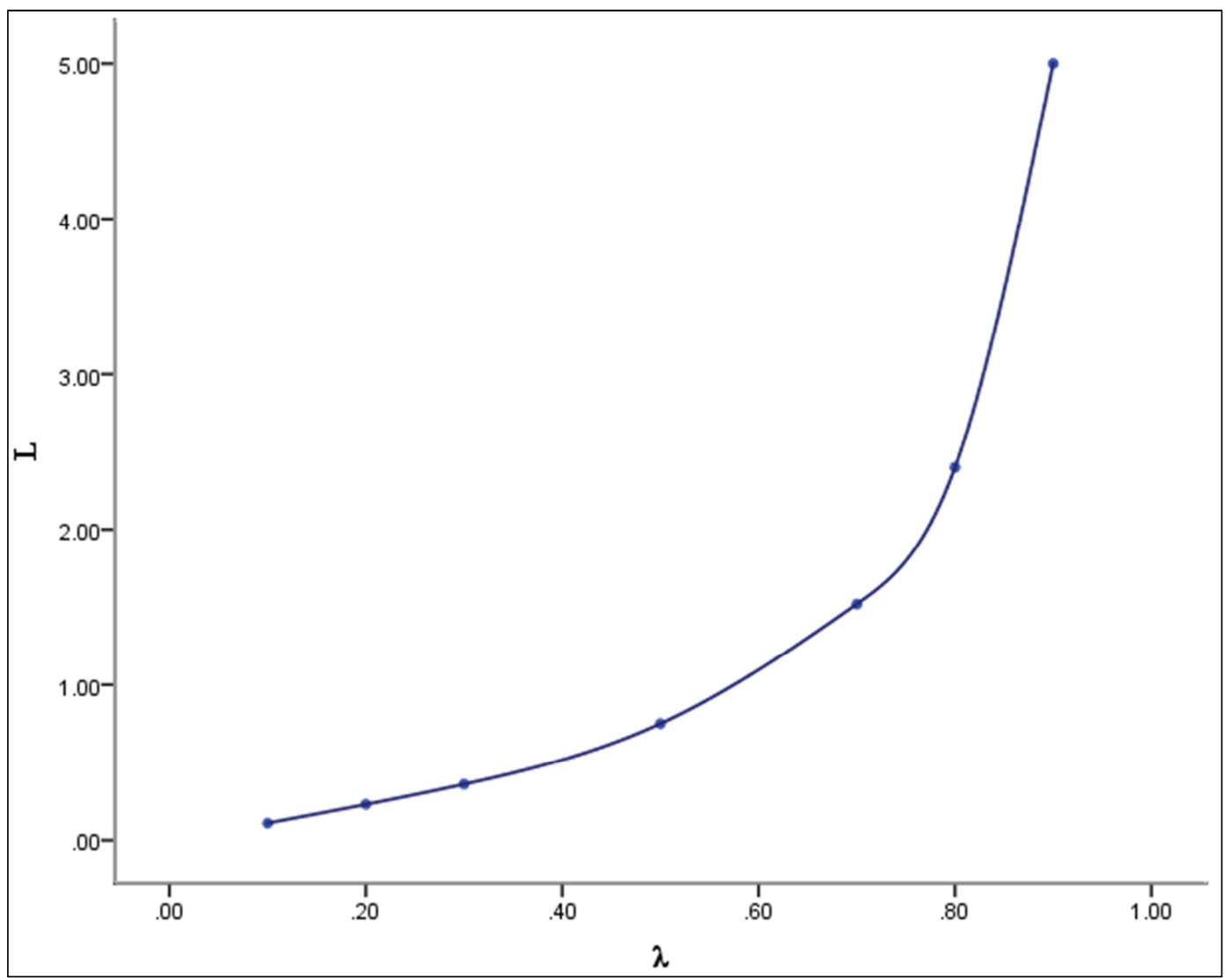

Figure 7. The relation between $L \& \lambda$

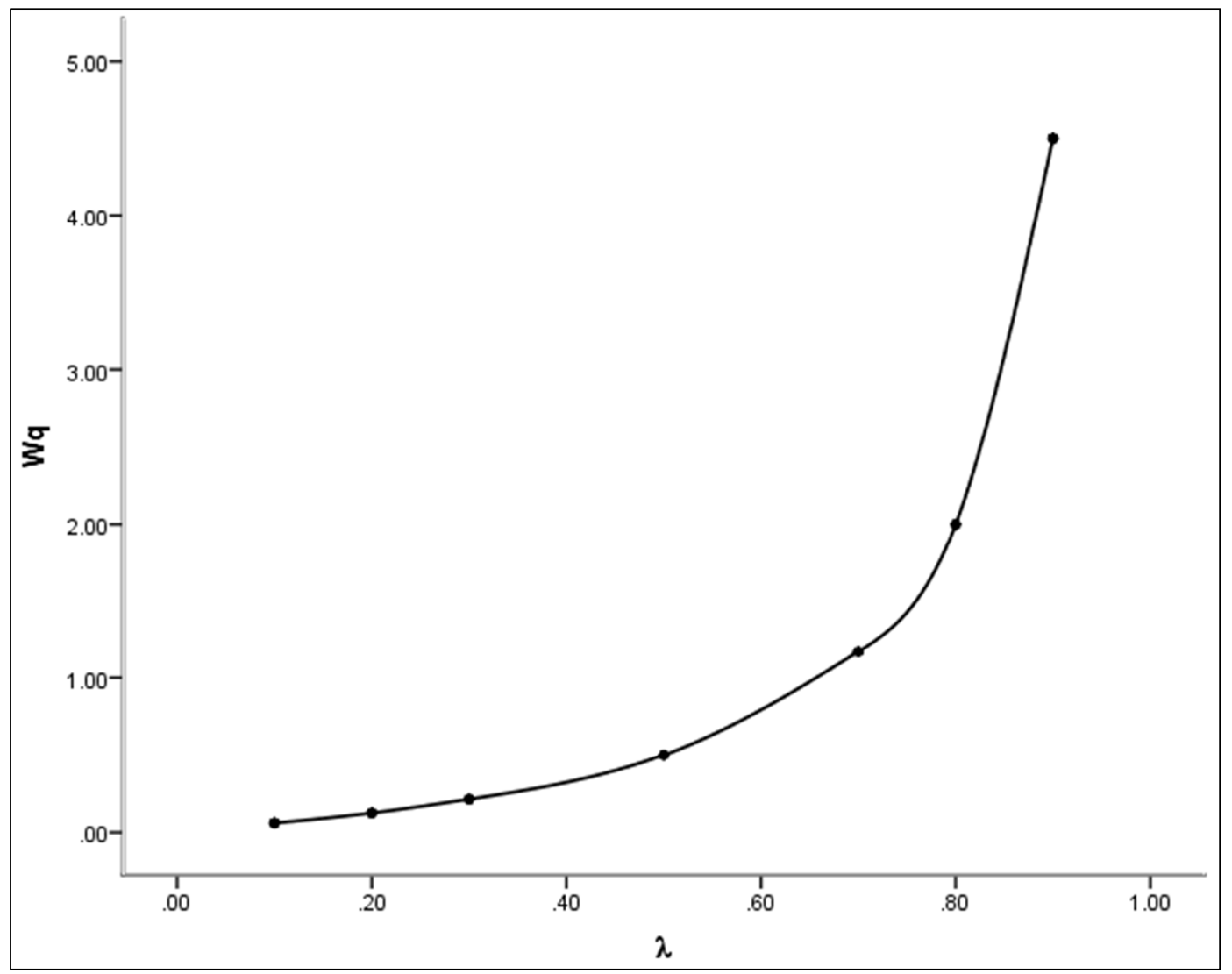

Figure 8. The relation between $W_{q} \& \lambda$. 
As figure 3, shows that the increased the arrival rate offset it decrease of the probability that there are no customers in the system. Also, from figures 4, 5 and 6, note all increased in arrival rate Offset by an increase and then decrease in the probability that there are $\mathrm{n}$ of customers in the system. And in figures 7 and 8 , shows that the increased the arrival rate offset it increase in the expected number of customers in the system and the queue.

\section{Conclusion}

This paper has explained the analytical solution in steadystate for $\mathrm{M} / \mathrm{D} / 1$ with addition the concept balking a probability generating function and iterative method were devised to determine the probability that there are $n$ customers in the system, the probability that no customers are in the service department, the expected number of customers in the system and the expected number of customers in the queue. Finally, the numerical example was confirmed to confirm the model.

\section{References}

[1] M. R. Oliver, Table of the waiting time distribution for the constant service queue $(\mathrm{M} / \mathrm{D} / 1)$, International journal computers mathematical, 2 (1968), 35-56.

[2] V. B. Iversen, Exact calculation of waiting time distributions in queueing systems with constant holding times, NTS-4, Fourth Nordic teletraffic Seminar, Helsinki (1982).

[3] V. B. Iversen and L. Staalhagen, Waiting time distribution in M/D/1 queueing systems, Electronics Letters, 35 (1999), 2184-2185.

[4] O. Brun and J. Garcia, Analytical solution of finite capacity
M/D/1 queues, Journal of applied probability, 4 (2000), 10921098 .

[5] E. V. Koba, Stability condition for $M / D / 1$ retrial queuing system with a limited waiting time, Cybernetics and systems analysis, 2 (2000), 184-186.

[6] E. V. Koba, An M/D/1 queuing system with partial synchronization of its incoming flow and demands repeating at constant intervals, Cybernetics and systems analysis, 6 (2000), 177-180.

[7] Kenji Nakagawa, On the series expansion for the stationary probabilities of an $\mathrm{M} / \mathrm{D} / 1$ queue, Journal of the operations research society of Japan, 2 (2005), 111-122.

[8] V. B. Iversen, Teletraffic engineering and network planning, Technical university of Denmark, (2007).

[9] D. Groos and C. M. Harris, Fundamentals of queueing theory, New York, John wiley and sons, 4th edition, (2008).

[10] K. L. Prasad and B. Usha, A comparison between M/M/1 and M/D/1 queuing models to vehicular traffic at Kannyakumari district, Journal of mathematics, 1 (2015), 13-15.

[11] M. I. Hussain, B. Ahmed and R. Ali, A discrete event simulation for the analytical modeling of $\mathrm{M} / \mathrm{D} / 1$ queues: Output buffer of an ATM multiplexer, Innovative Computing Technology (INTECH), (2016).

[12] B. Kim, J. Kim, Explicit solution for the stationary distribution of a discrete-time finite buffer queue, Journal of Industrial and Management Optimization, 12 (2016), 11211133.

[13] J. W. Baek, H. W. Lee, S. Ahn and Y. H. Bae, Exact timedependent solutions for the $\mathrm{M} / \mathrm{D} / 1$ queue, Operations Research Letters, 44 (2016), 692-695.

[14] Kotobi and Bilén, Spectrum sharing via hybrid cognitive players evaluated by an M/D/1 queuing model, Journal on wireless communications and networking, 85 (2017), 1-11. 